\section{Best management of irritable bowel syndrome}

Irritable bowel syndrome is common and can have a considerable impact on quality of life. Early diagnosis and timely and evidence based management is key to improved outcomes and best management. Consideration of the differential diagnosis is important. Christopher Black and Alexander Ford have previously written for us on rational and appropriate investigation. ${ }^{1}$ In this issue the authors cover 'Best Management'. Multiple different treatment options are discussed including anti-spasmodics, peppermint oil, neuromodulators and tricyclics. There is a strong focus on good communication and holistic care and setting realistic expectations of treatment. Simple lifestyle and dietary strategies are important. A low FODMAP (fermentable oligosaccharides, disaccharides, monosaccharides and polyols) can be considered providing dietetic support is available. Patients with constipation resistant to laxatives should be offered a trial of linaclotide. For patients with diarrhoea that fails to respond to loperamide alosetron and ramosetron can be considered. For patients who are symptomatic with significant impact on their functioning cognitive behavioural therapy and gut directed hypnotherapy should be considered both of whom have a reasonable evidence base. Refractory IBS can be very challenging. This is an excellent review of the best available treatment options evidence based, focused and practical for implementation by the busy clinician. Listen to the linked Podcast. Editor's Choice this month (See page 303).

\section{Real-World evaluation of an intravenous iron service for the treatment of iron deficiency in patients with gastroenterological disorders}

Iron deficiency anaemia is common in gastroenterological disorders including inflammatory bowel disease although not always straightforward to treat particularly in the context of active gut inflammation. There has been an increase in the use of intravenous iron in inflammatory bowel disease (IBD) with clinical guidance from the European Crohn's and Colitis Organisation (ECCO) recommending intravenous iron for patients with iron deficiency anaemia in the context of clinically active disease. In this issue Kearns and colleagues report their 'real world' experience as part of a service evaluation and improvement process looking at estimated iron need vs dose, delivery, safety and outcome. 117 patients are reported (including 51 with IBD). There were a wide variety of non-IBD conditions reflecting the reality of clinical practice. Most patients $(76.1 \%)$ received their estimated iron need $(62.4 \%)$ in one appointment. Mean (SD) dose was 1317 (409.7) mg. No serious adverse effects were seen. Patients who received less than their estimated iron need were more likely to be anaemic at 6 months. Mean $\mathrm{Hb}$ increased from $101.6 \mathrm{~g} / \mathrm{L}$ at baseline to 122.8 at first follow-up around 1 month and $124.2 \mathrm{~g} / \mathrm{L}$ at 6 months although ferritin levels fell by 6 months (data is in the paper). The authors confirm safety and efficacy, the importance of adequate dosing and the need for follow-up monitoring of the haemoglobin and iron status. Important paper reflecting the effective local implementation of national and international guidance (See page 265).

\section{Gastrointestinal manifestations of COVID-19 in children: a systematic review and meta- analysis}

There have been many excellent publications on COVID-19 pulling information together to inform management in parallel to the ongoing pandemic. In this issue Akobeng and colleagues report a systematic review and meta-analysis of the gastrointestinal manifestations of COVID-19 in children - initially published online in August 2020 - 13 case series, four case reports, 284 patients. In keeping with adult data gastrointestinal symptoms are common, present in up to $25 \%$ and include diarrhoea, vomiting and abdominal pain. This early data was an important part of the evidence base highlighting the non- respiratory manifestations and the importance of atypical presentations of COVID-19 (See page 332).

\section{Disease monitoring of biologic treatment in IBD: early impact and future implications of COVID-19 pandemic}

COVID-19 has forced us to reflect on our practice and make practical changes to enable us to do the best for our patients within the limitations enforced by the ongoing pandemic. In this issue Shields and colleagues consider this - published on line in September 2020 - discussing the secondary impact of COVID-19 on patients with inflammatory bowel disease. This has included less access to acute services, less endoscopy, temporary cessation of faecal calprotectin measuring because of the risk of transmission and a significant reduction in therapeutic drug monitoring. The authors summarise their practice adaptations - listed in full in the article and including increase use of virtual technology, expansion of capacity of the helpline, 'hot' IBD clinics, cohorting of biological infusions, phlebotomy clinics for selected patients and the launch of remote point of care faecal calprotectin testing. As the pandemic starts to settle, at least in the UK, we need to plan for a post COVID-19 world and continue with at least some of the positive changes which have helped stream line care and deliver services more effectively for the benefit of patients (See page 345).

\section{Challenges and opportunities of COVID-19 for gastroenterology and hepatology services}

The opportunity during the recovery phase of the pandemic to 're boot and redesign' our services including the way we work across the gastroenterology and hepatology is considered further by Tham and colleagues in an excellent opinion piece which includes six key principles for service redesign enhanced senior triage, enhanced team working, the correct balance between virtual and face to face consultations, an enhancement of the 'one stop' approach to consultations, reliable and dynamic information technology and pro-active job planning to recognise change and innovation. The practicalities of implementation of these strategies will require dynamic local and national leadership and have the potential - post pandemic - to enhance the care offered to patients (See page 342).

\section{How can gastroenterology training thrive in a post-COVID world?}

The impact of COVID-19 has been massive for education and training 


\section{UpFront}

in all sectors and the recovery from this is going to be long and complex. This presents different challenges in different areas. In this issue Fitzpatrick and colleagues, in an opinion piece, discuss the practicalities for gastroenterology training - increasingly craft based - in the context of the implementation of shape of training. The ongoing constraints are well known and continue to change in parallel to the ongoing pandemic. The authors carefully work through the challenges and propose solutions focussing on endoscopy training, training in clinic, subspecialty exposure and planned education activities. The strategies will of course evolve but this is an important document to read now. The trainees reflect the future of our specialty and this article is forward thinking and dynamic in its approach remembering that 'out of adversity comes opportunity' (See page 338).
COVID-19 and the challenges

faced by gastroenterology trainees: time for capsule endoscopy training?

There is no doubt the impact on endoscopy services has been profound from a service and training point of view with now a massive backlog of cases. In this issue Conley and colleagues discuss this reflecting on the potential 'window' to develop further the role of capsule endoscopy in our clinical practice and consider the training opportunities and practicalities. This will require a clear structure and certification process with the establishment of service delivery and training networks. The enhanced information technology necessitated by COVID-19 and potential for remote reporting are fundamental to this being effective. In an excellent accompanying commentary Mark McAllindon discusses the huge future potential for capsule endoscopy including the application of artificial intelligence to enhance diagnostic provision
- COVID-19: impetus to the adoption of capsule endoscopy as a primary diagnostic tool? (See pages 299).

Please enjoy this issue. Please continue to read, enjoy and feedback on the journal. Follow us on twitter@FrontGastro_BMJ and listen to our regular podcasts accessed via the journal website https://fg.bmj.com/

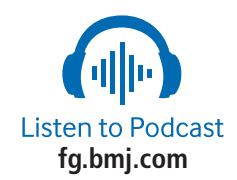

ORCID iD

R Mark Beattie http://orcid.org/00000003-4721-0577

\section{REFERENCE}

1 Black CJ, Ford AC. Rational investigations in irritable bowel syndrome. Frontline Gastroenterol 2020;11:140-7. 